\title{
La Géomorphologie De La Plage De Haouzia (Littoral Atlantique Marocain) : Origine Et Mise En Place De Ses Sédiments Actuels
}

\author{
Soukaina Khouaja \\ Mohamed Ouadia \\ El Mehdi Irzan
}

Laboratoire des Géosciences et Techniques de l'Environnement, Département de Géologie, Faculté des Sciences, Université Chouaib Doukkali. Route Ben Mâachou, El Jadida, Maroc

doi: 10.19044/esj.2016.v12n24p253 URL:http://dx.doi.org/10.19044/esj.2016.v12n24p253

\begin{abstract}
Geomorphological study of the range of Haouzia shows that the latter is more or less homogeneous but influenced by the mouth of the Oued Oum Rbia to the No. Spatio-temporal variation and the heterogeneity and beach profiles are related to the morphology of the beach and the coastal dynamics. Its sediments enrich carbonate because they feed Cretaceous land upstream of the mouth and the Quaternary calcarenites outcropping in the submerged area of the beach. Heavy minerals come from land metamorphic (Boulaouane) and magmatic (Sidi Saïd Maachou) through the Oued Oum Rbia and then taken over by the littoral drift. The weight content of heavy minerals is a good indicator of the direction of the transfer (no SW) of these minerals and sediment. The relative heterogeneity of sediments is favoured by the mouth of the Oued Oum Rbia allowing mixing of continental and marine sediments. It is all the more pronounced to the SW (breaking the waves) and winter (storms). Sediment (coarse and fine) are generally ranked especially in part SW (quiet environment despite the onslaught of the waves) and dune (sorting by the wind). Haouzia beach is under anthropogenic pressure to order, essentially, tourist who is behind the deforestation of the forest of Hameed and therefore the destabilization of the environment (including the beach). Urgent action is needed to protect this beach playing an important socio-economic role in the region.
\end{abstract}

Keywords: Haouzia, beach, geomorphology, sedimentology, mineral grains, anthropic action evolution, space-time, mouth of the Oued Oum Rbia 


\section{Résumé}

L'étude géomorphologique de la plage de Haouzia montre que cette dernière est plus ou moins homogène mais influencée par l'embouchure de l'Oued Oum Rbia, plus particulièrement, au NE. La variation spatiotemporelle et l'hétérogénéité des profils de plage sont liées à la morphologie de la plage et à la dynamique littorale. Ses sédiments s'enrichissent en carbonates à partir des terrains crétacés en amont de l'embouchure et des calcarénites quaternaires affleurant dans la zone immergée de la plage. Les minéraux lourds proviennent des terrains métamorphiques du Paléozoique (Boulaouane) et magmatiques du PérmoTrias (Sidi Said Maachou) par l'intermédiaire de l'Oued Oum Rbia et sont repris par la dérive littorale. La teneur pondérale de ces minéraux lourds est un bon indicateur du sens du transfert (NE vers SW) de ces minéraux et des sédiments. L’hétérogénéité, relative, des sédiments est favorisée par l'embouchure de l'Oued Oum Rbia permettant le brassage de sédiments continentaux et marins. Elle est d'autant plus prononcée au SW (déferlement des vagues) surtout en hiver (tempêtes). Les sédiments (grossiers et fins) sont en général bien classés surtout dans la partie SW (déferlement des vagues) et dans la dune (tri sélectif par le vent). La plage de Haouzia est sous pression anthropique d'ordre- essentiellement, touristique qui est derrière le déboisement de la forêt de Haouzia, en plus du dragage des sables de l'embouchure et par conséquent la déstabilisation du milieu (y compris la plage). Une intervention urgente s'impose pour protéger cette plage qui joue un rôle socioéconomique non négligeable dans la région.

Mots clés : Haouzia, plage, géomorphologie, sédimentologie, minéraux en grains, action anthropique, évolution, spatiotemporelle, embouchure de l’Oued Oum Rbia

\section{Introduction}

La plage sableuse de Haouzia, qui est l'objet de ce travail, représente une grande baie entre les villes d'Azemmour et El Jadida. Sa morphologie et ses dépôts sont influencés par l'embouchure de l’Oued Oum Rbia située au niveau de Azemmour.

Dans ce travail, on essaye de mettre en relief les caractéristiques morphologiques, sédimentologiques ainsi que la pression anthropique que subit cette plage, en particulier, au cours de ces dernières années. Cette étude permet, également, de discuter les divers facteurs agissant sur ce milieu, sur ses profils et sur son évolution spatiotemporelle. Elle a aussi pour but de comprendre les conditions de mise en place des sables de cette plage, leur 
origine et le sens de leur transit le long du littoral. Elle permet, également, de suivre l'interaction du fleuve Oum Rbia et de la mer le long de cette plage.

\section{Situation géographique et caractéristiques du site}

La plage de Haouzia fait partie de la côte atlantique marocaine, elle est limitée au Nord-Est par la ville d'Azemmour, au Sud-Ouest par la ville d'El Jadida et à l'Est par la zone touristique. Ses coordonnées Lambert sont : $33^{\circ} 19^{\prime} 00.96^{\prime \prime}$ 'Nord / $8^{\circ} 20^{\prime} 23.17^{\prime \prime}$ Ouest et $33^{\circ} 14^{\prime} 44.23^{\prime \prime}$ Nord / 8²7’44.22'’Ouest.

C'est une plage allongée NE-SW. Elle s'étend sur une distance de l'ordre de $14 \mathrm{~km}$, avec l'embouchure de l'Oued Oum Rbia au NE et la falaise côtière de la plage de Deauville au SW. Très peu de rochers affleurent dans sa partie immergée lors de la basse marée. La dune qui la borde du coté du continent est très développée au NE par rapport au SW (Akil M., 1990). L'accès à la plage se fait, d'une manière clandestine hasardeuse à cause de l'absence de l'infrastructure. Transversalement à la côte, la plage est composée de l'estran, du haut de plage et d'une dune composée de dunes embryonnaires et d'autres plus ou moins fixées. Ces dernières années, plusieurs constructions commencent à voir le jour dans cette zone ainsi que plusieurs parkings mal repartis et non équipés (Fig. 1).

Figure 1 : Situation géographique de la plage de Haouzia (Photo Google Earth).

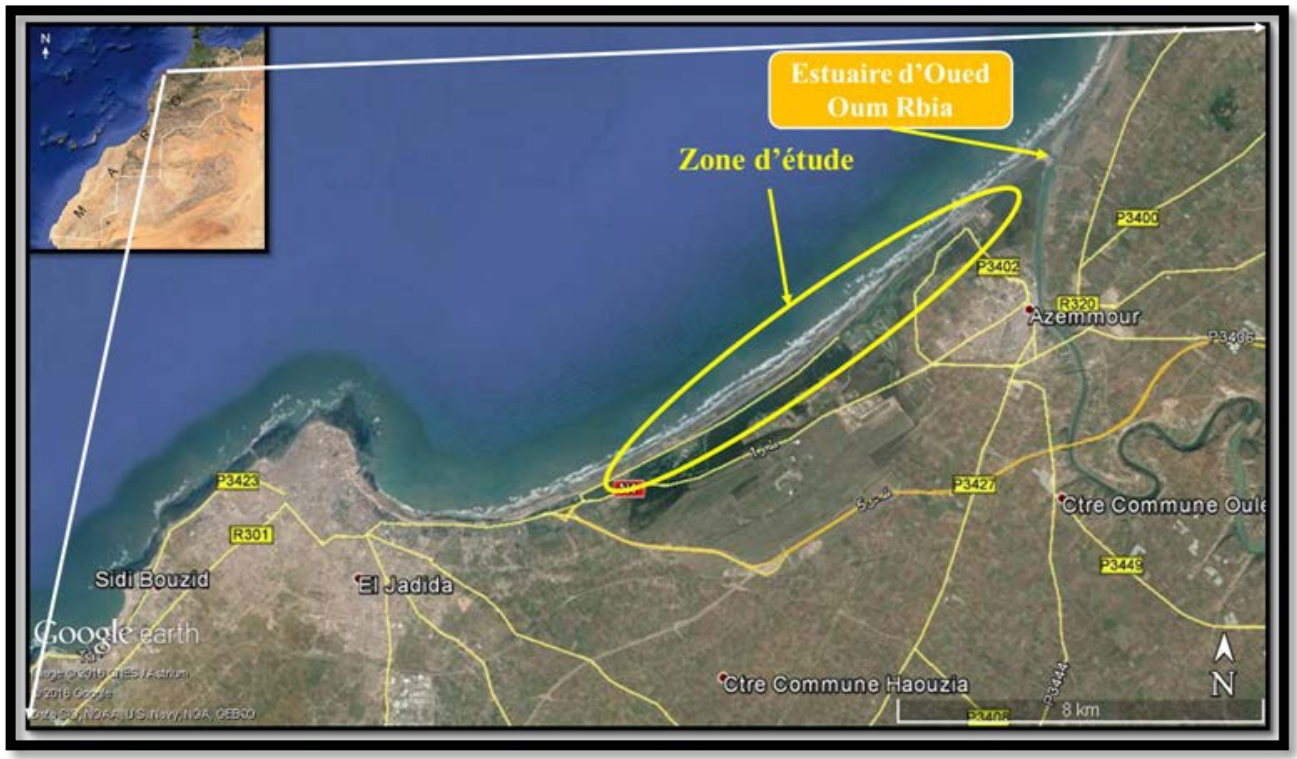

\section{Matériel et méthodes}

Sur le terrain, des relevés des profils de plage ont été faits, en deux missions (hiver et été), mais également un échantillonnage (neuf échantillons par mission) parallèlement et perpendiculairement à la côte (trois radiales : 
au NE, au centre et au SW de la plage). Ce dernier concerne les sédiments de l'estran, du haut de plage et de la dune.

Une étude granulométrique (selon les normes d'AFNOR) et minéralogique (minéraux lourds : selon la méthode de (Parfenoff A. et al., (1970)) a été faite pour chaque échantillon.

Concernant la granulométrie, on s’est intéressé à la fraction 1,250,050 mm, préalablement attaquée par l'acide chlorhydrique puis, rincée, séchée, pesée et tamisée (colonne de 11 tamis). On pèse le refus de chaque tamis et on traite les données pour déduire des pourcentages simples et cumulés qui serviront à tracer des courbes granulométriques. Ces dernières permettent de déduire des paramètres et de calculer des indices granulométriques (Vatan A., 1967).

Les minéraux lourds sont étudiés, quantitativement et qualitativement, après leur séparation en fonction de leur densité (grâce au Bromoforme : $d=2,89$ ) suivie par leur détermination. Les graphiques tracés permettant de comparer et de suivre l'évolution spatiotemporelle de ces minéraux.

La calcimétrie a été également réalisée (attaque totale des carbonates par l'acide chlorhydrique) pour évaluer le taux total des carbonates dans les échantillons.

\section{Résultats}

\section{Etude géomorphologique}

\section{Morphologie de la plage}

Jusqu'à notre jour, aucune étude, associant à la fois la géomorphologie, la sédimentologie et la minéralogie des sédiments, de la plage de Haouzia n’a été faite auparavant à l'exception de celle des profils et de la dynamique (Chaibi M., 2003). C'est pour cela que nous essayons de souligner les grands traits qui caractérisent cette plage. En effet, cette plage est bordée vers l'Est par une dune complexe et, plus ou moins, fixée

Cette plage abrite au niveau de sa dune de grandes infrastructures hôtelières. Au NE, elle est limitée par l'estuaire de l'Oum Rbia et au SW par les premières falaises côtières qui apparaissent après une longue distance de plage sableuse. La forme de la plage est presque rectiligne et étroite dans ses deux extrémités et large au centre. Peu de rochers affleurent dans la partie immergée de la plage. Ces derniers sont formés de conglomérats fluviomarins quaternaires) consolidés au NE et de calcarénites au centre et au SW (Ouadia M., 1998). Le couvert végétal au niveau de la dune est plus ou moins développé surtout au NE et commence à devenir moins développé et dégradé vers SW. Les vagues montrent un léger déferlement en particulier au SW de la plage. 
L'étude de la comparaison des deux photos Google Earth (2004 et 2016) prises pendant les mêmes saisons (printemps) reflète que la dynamique marine ait un impact sur la morphologie de la baie de Haouzia. Au NE, c'est la sédimentation au niveau du haut de plage qui domine. Ces constatations sont prouvées par l'évolution de la baie montrant une tendance au rétrécissement de sa zone $\mathrm{SW}$ et au développement de sa zone $\mathrm{NE}$ et au centre. Pour estimer la tendance à l'élévation du niveau marin, on a calculé la surface de la plage qui commence à être influencée par l'action de la mer. Elle est de l'ordre de 36 m (en douze ans : 2004-2016).

\section{Profils}

Les profils de la plage de Haouzia sont hétérogènes et montrant une variation spatiotemporelle. Ils sont relativement plus développés au NE par rapport au SW de la plage (Fig. 2). L'écart, le plus important, de l'altitude de ces derniers est celui du SW et au centre de la plage. Les profils de l'été sont, en général, plus hauts et paraboliques (accrétion) que ceux de l'hiver en particulier au NE et au centre de la plage. La berme est plus individualisée en hiver surtout pour le secteur NE. C'est le seul endroit où elle est matérialisée dans un estran à galets de la rive gauche de l'embouchure de l'Oued Oum Rbia. Le centre bénéficie d'une sédimentation importante en été plus que les autres secteurs surtout au niveau du haut de plage (Fig. 3).

Figure 2 : Localisation des profils de la plage de Haouzia.

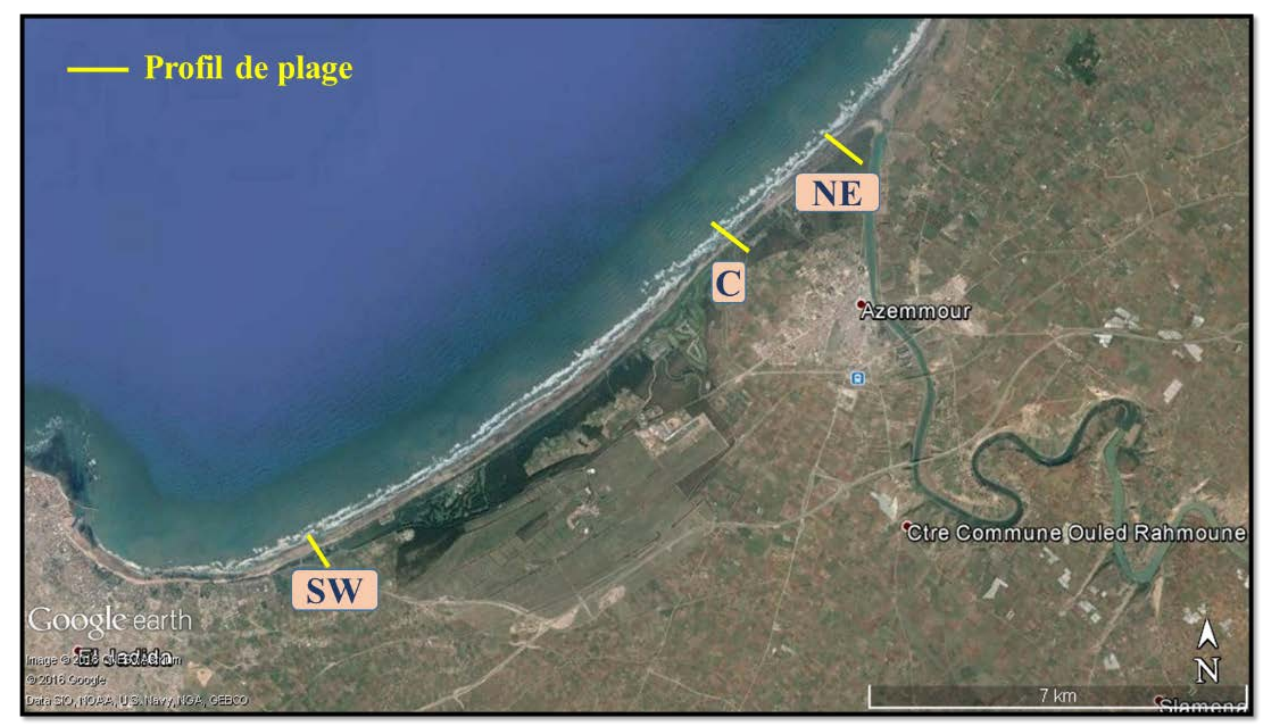




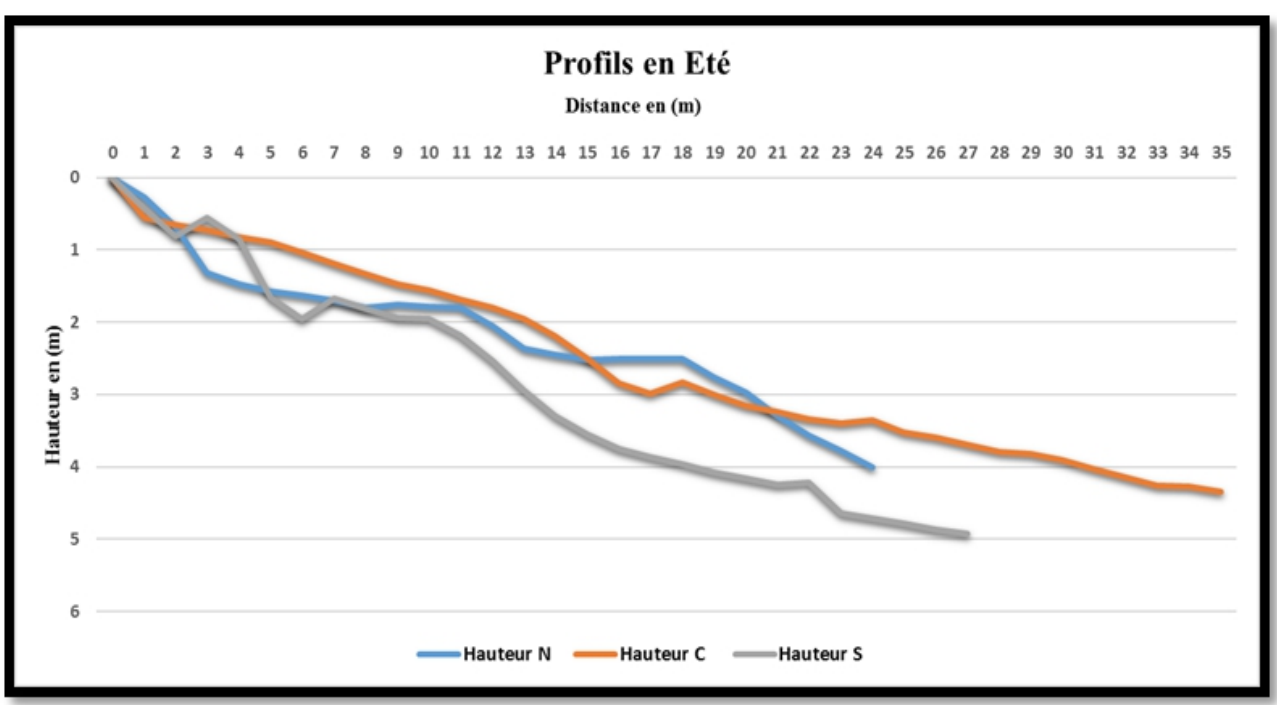

Figure 3 : Les profils (en été) de la plage de Haouzia.

\section{Etude sédimentologique}

\section{Calcimétrie}

Le taux des carbonates est en général moyen. Au NE, il est moins élevé par rapport au SW. Cependant, perpendiculairement à la plage, les sédiments de l'estran et du haut de plage sont relativement riches en carbonates par rapport aux sédiments de la dune. Concernant la variation saisonnière, ce taux est plus élevé en hiver qu'en été (Fig. 4).

Figure 4 : Evolution des taux des carbonates dans les sables de la plage de Haouzia.

(H : Haouzia ; Es : estran ; HP : haut de plage ; D : dune)
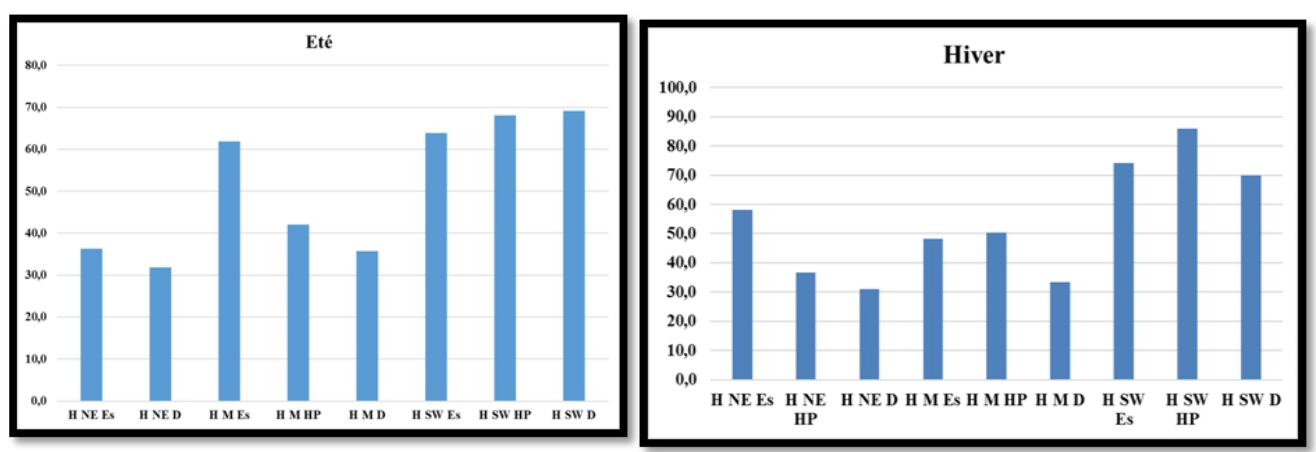

\section{Granulométrie}

\section{Courbes granulométriques}

Quelques échantillons analysés présentent des courbes granulométriques simples polymodales. Le nombre de mode élevé est enregistré dans les sédiments au SW plus que dans ceux du NE, et aussi dans les sédiments de l'estran et du haut de plage plus que dans ceux de la dune. 
Ce nombre est aussi important dans les sédiments de l’hiver en estran et dans ceux de la dune en été (Fig. 5).

Figure 5 : Evolution saisonnière des modes des sables de la plage de Haouzia.
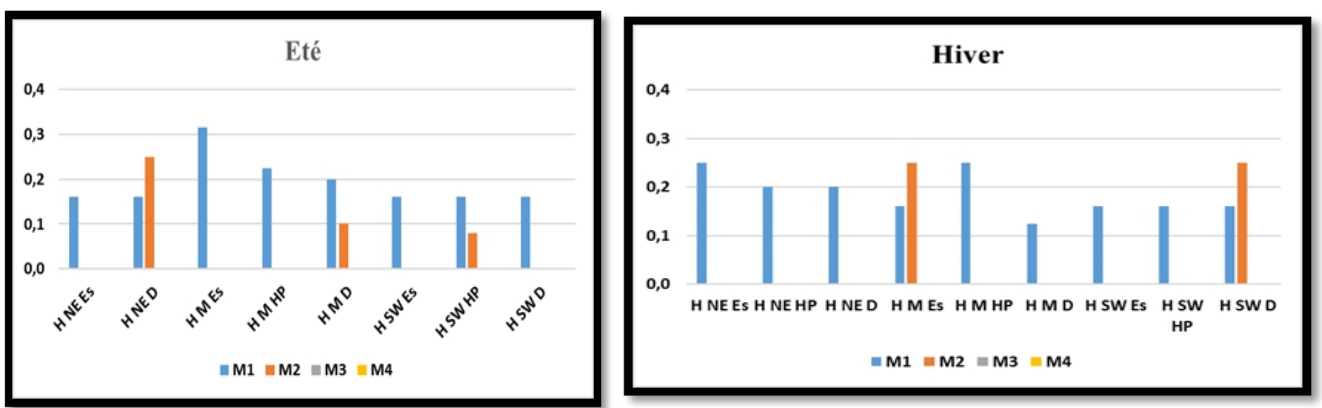

\section{Paramètres granulométriques}

Les paramètres granulométriques montrent, en général, une légère diminution du NE au SW. Transversalement à la plage, les valeurs des paramètres granulométriques des sédiments de l'estran et du haut de plage sont élevées par rapport à celles des sédiments de la dune. Les valeurs des paramètres granulométriques montrent relativement une diminution en été par rapport à l'hiver (Fig. 6).

Figure 6 : Evolution des paramètres granulométriques (Q1, Q2 et Q3).
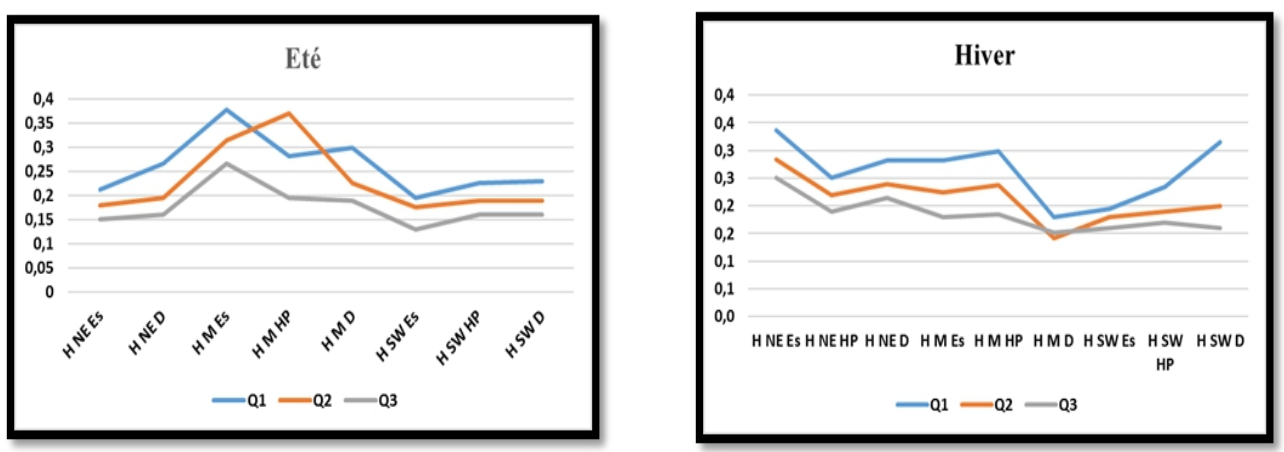

\section{$>\quad$ Indices granulométries}

- $\quad$ Sorting index (So)

So oscille, en général, entre 2,1 et 2,3. On ne note pas un grand changement du NE au SW. Par contre, une légère diminution de So est remarquable en allant de l'estran vers la dune.

Quant aux variations saisonnières, les valeurs de So de l'été ne différent pas beaucoup de celles de l'hiver.

- $\quad$ Skewness (Sk)

Sk est compris entre 0,4 et 1,4. Du NE au SW, les valeurs de Sk augmentent légèrement. Perpendiculairement à la plage, les valeurs de Sk 
augmentent en allant de l'estran vers la dune à l'exception du haut de plage au centre et en été où se sont enregistrées des valeurs faibles de Sk. Les valeurs de Sk montrent, généralement, une légère diminution en l'été par rapport à l'hiver (Fi.g 7).

Figure 7 : Evolution des indices granulométriques (So et Sk).
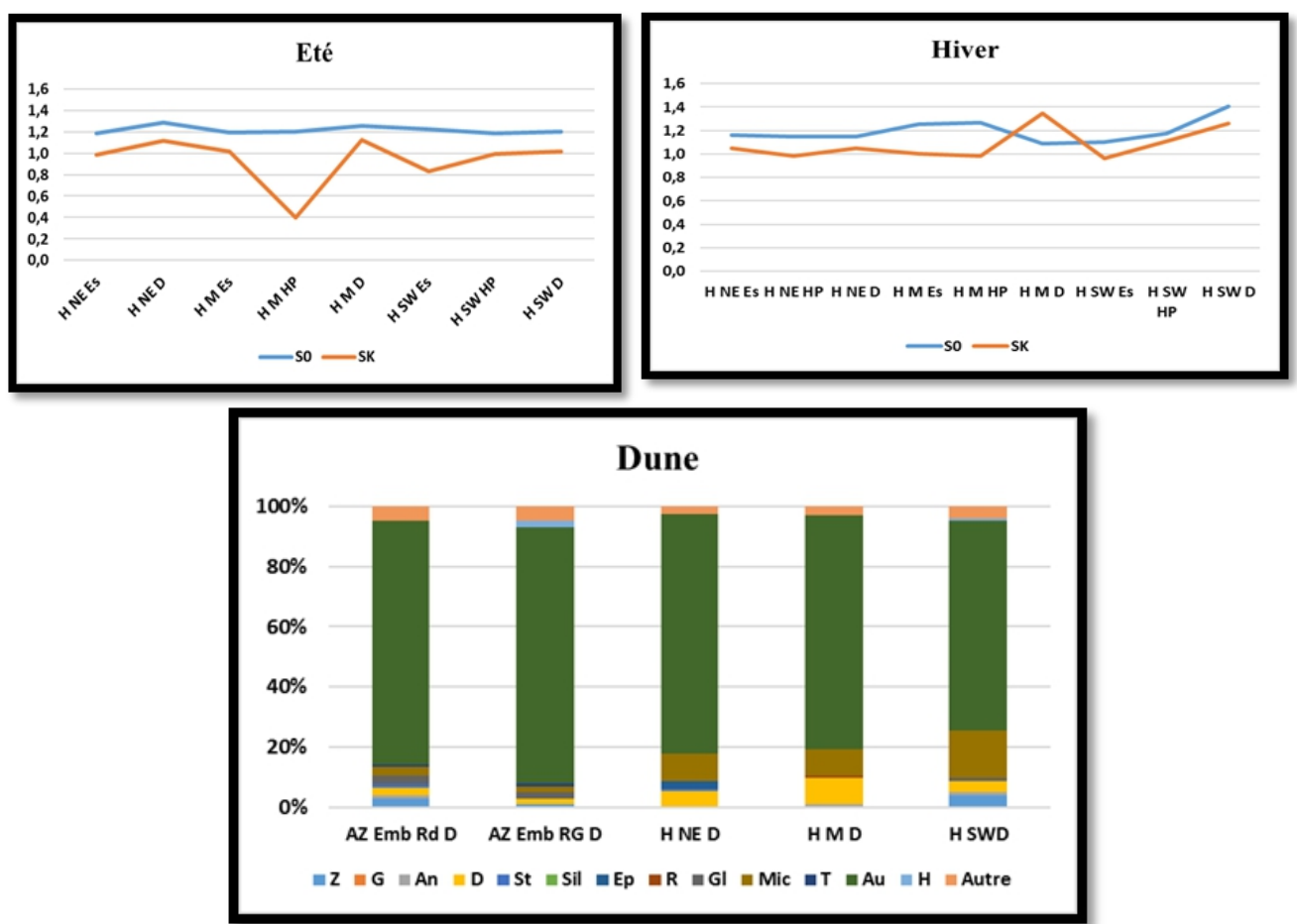

\section{Etude minéralogique (minéraux lourds)}

Les minéraux lourds des sédiments de la plage de Haouzia présentent un cortège minéralogique varié, quantitativement et qualitativement, prédominé par ordre décroissant par : le pyroxène suivi par les micas, puis par le disthène. Une tendance à l'augmentation du taux des micas, contrairement à celui du pyroxène, est remarquable, en particulier dans la dune, du NE au SW. On note, également, une tendance à l'enrichissement des sédiments de la dune en pyroxène par rapport à ceux de l'estran et du haut de plage.

La teneur pondérale des minéraux lourds est contrastée le long de la plage $(20,86 \%$ : dune au NE prés de l'embouchure et seulement de $1 \%$ : estran au centre de la plage) (Fig. 8). 

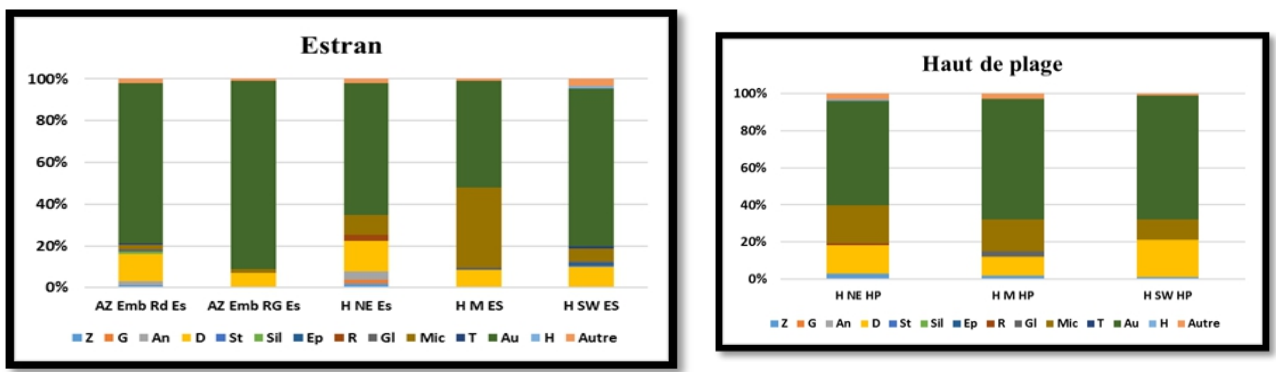

Figure 8 : Evolution du cortège des minéraux lourds des sédiments de la plage de Haouzia (Z : Zircon ; G : Grenat ; An : Andalousite ; D : Disthène ; St : Staurotide ; Sil : Sphène ; Ep : Epidote ; R : Rutile ; Gl : Glauconite ; Mic : Micas ; T : Tourmaline ; Au : Augite ; H : Hornblende).

\section{Action anthropique}

La zone de Haouzia est récemment très demandée par des investisseurs dans le domaine du tourisme. Des chaînes hôtelières et un grand salon national d'exposition viennent d'être construits en plus d'une ville verte qui est planifiée. Toutes ces constructions sont faites aux dépens, en partie, de la forêt avoisinant la plage de Haouzia. Vu son ampleur, la plage de Haouzia attire plus d'estivants alors qu'aucune infrastructure n'est faite auparavant. D'où une prolifération des accès hasardeux à la plage et des parkings clandestins qui conduisent au déboisement progressif de la forêt et à la fragilisation de la dune (Fig. 9).

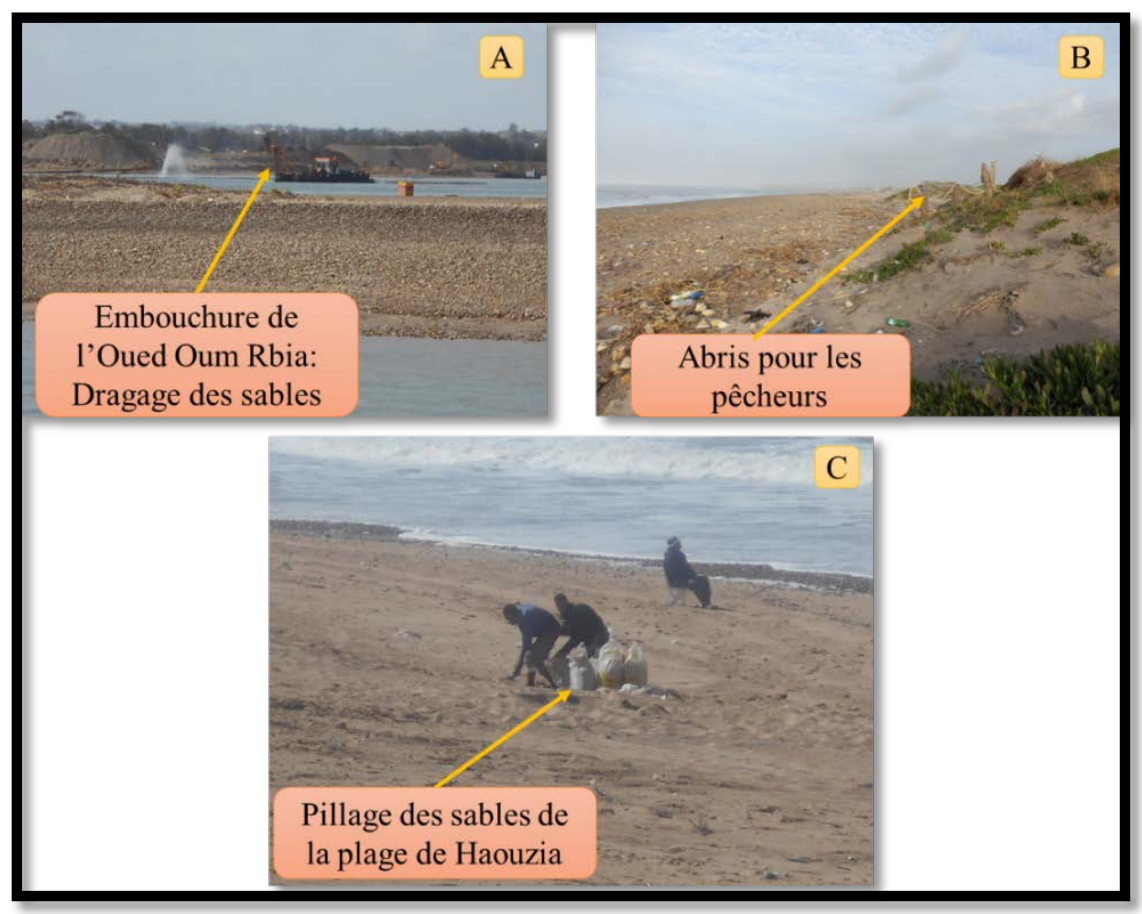

Figure 9 : Action anthropique 


\section{Interprétation et discussion}

\section{Etude géomorphologique}

\section{Morphologie de la plage}

L'étude géomorphologique de la plage de Haouzia montre que cette dernière a connu, ces dernières années, des changements radicaux sur le plan d'aménagement et de tourisme. Cette étude montre qu'il s’agit d'une plage, quoique ample, fragile et ne cesse de se dégrader. Elle est bordée par deux zones en pleine mutation : l'embouchure de l'Oum Rbia au NE et la dune en voie de dégradation à l'Est. La forme de la plage est rectiligne mais plus large au centre. Les falaises la limitant au SW sont à l'origine de l'agressivité de la dynamique marine. Peu de rochers affleurent dans la partie immergée de la plage. Sur la dune, le couvert végétal est relativement développé au NE plus qu'au SW. Il y a plus de déferlement de vague au SW par rapport au NE. Avec le temps (2004-2016), la comparaison des Photos Google Earth révèle une extension de la zone, sous l'influence des marées, de 36 m (en douze ans) en témoignant d'une tendance à l'élévation du niveau marin et, par conséquent, probablement au réchauffement climatique.

\section{Profils}

Les caractéristiques et l'hétérogénéité des profils de plage révèlent que la morphologie de la plage et l'interaction des dynamiques marine et éolienne ont influencé de manière très significative cette plage de Haouzia.

\section{Etude sédimentologique}

L’étude sédimentologique de la plage de Haouzia et de la rive gauche de l'Oued Oum Rbia, entamée dans ce travail, vient compléter quelques études limitées, faites auparavant, qui ne touchaient que l'embouchure de l'Oued Oum Rbia (Zourarah B., 2002) et essentiellement les dunes de Haouzia (Chaibi M. et al., 2010). Elle actualise et complète d'autres études (Aboumaria Kh., 1993, Mhammdi N. et al., 2005, Aberkan M. et al., 2014 et 2015, Ouadia M. et al., 2015 et Khouaja S. et al., 2016 b) qui intéressaient quelques zones avoisinantes.

\section{Calcimétrie}

Les carbonates alimentant les dépôts de plage de Haouzia proviennent essentiellement du substratum calcarénitique quaternaire (Khouaja S. et al., 2016 a) mais aussi des calcaires crétacés le long de l’Oued Oum Rbia. Cependant, le taux de ces carbonates qui est moyen s'explique par la dilution des sédiments de la plage par des dépôts terrigènes apportés par l'Oued Oum Rbia d'où les faibles taux des carbonates au NE à proximité de l'embouchure de l'Oum Rbia. Cependant, perpendiculairement à la plage, les sédiments de l'estran et du haut de plage sont relativement riches en 
carbonates par rapport à ceux de la dune et c’est grâce au tri sélectif du vent. Concernant la variation saisonnière, ce dernier est élevé en hiver plus qu'en été à cause, une autre fois, de l'agressivité de la dynamique marine et par conséquent d’une érosion importante du substratum calcaire.

\section{Granulométrie}

\section{$>\quad$ Courbes granulométriques (simples et cumulées)}

La polymodalité de certaines courbes granulométriques simples témoigne de l'hétérogénéité des sédiments de quelques échantillons en particulier au SW de la plage mais aussi dans l'estran et le haut de plage plus que dans la dune et ce à cause du déferlement des vagues ainsi qu'à l'irrégularité de la dynamique marine. L’hétérogénéité des sédiments de l'estran pendant l'hiver s'explique par l'action des tempêtes, et dans la dune en été par l'intensité et les fluctuations des directions du vent.

\section{$>\quad$ Paramètres granulométriques}

La légère diminution des paramètres granulométriques du NE au SW traduisent une tendance à la faiblesse de l'énergie marine au SW. Transversalement à la plage, les valeurs des paramètres granulométriques des sédiments de l'estran et du haut de la plage sont élevées vis-à-vis de celles des sédiments de la dune et témoignent de la forte dynamique marine par rapport à la dynamique éolienne. Les valeurs des paramètres granulométriques montrent une diminution relative en été par rapport à l'hiver qui traduit l'impact des fortes tempêtes en hiver sur la dynamique marine.

\section{$>\quad$ Indices granulométriques}

- $\quad$ Sorting index (So)

Les valeurs de So inférieures à 2,5 prouvent, en général, un bon classement des sédiments. Celles-ci tendent à diminuer de l'estran vers la dune à cause du bon classement des sédiments éoliens vis-à-vis ceux de l'estran et du haut de plage. Quant aux variations saisonnières et du NE au SW, elles ne montrent pas un grand changement.

- $\quad$ Skewness (Sk)

Les valeurs de Sk, allant de 0,4 à 1,4, traduisent que le classement est tantôt du côté des sédiments grossiers, tantôt du côté des sédiments fins. Au SW et de l'estran vers la dune, à l'exception du haut de plage au centre et en été, les valeurs de Sk augmentent légèrement. Elles témoignent du degré relativement fort de la dynamique. Les valeurs de Sk montrant, généralement, une légère diminution en été par rapport à l’hiver traduisent la tendance à la faiblesse de la dynamique pendant l'été. 


\section{Etude minéralogique (minéraux lourds)}

Le large spectre des minéraux lourds et leur variation quantitative et qualitative témoignent de la diversité de leur origine. Le pyroxène suivi par les micas puis par le disthène prédominent les autres minéraux, ce qui reflète que se sont essentiellement les terrains métamorphiques et magmatiques qui alimentent la plage de Haouzia par l'intermédiaire de l’Oued Oum Rbia puis par la dérive littorale. Les premiers (quartzites paléozoïques) affleurent en abondance à Boulaouane et les seconds (basaltes pérmo-triasiques) à Sidi Said Maachou.

La teneur pondérale des minéraux lourds élevées au NE (près de l'embouchure) témoigne du rôle de cet Oued dans l'acheminement des minéraux de l'arrière-pays vers la plage de Haouzia.

\section{Action anthropique}

La plage de Haouzia est fortement influencée par l'action anthropique. Cette dernière réside dans les investissements importants sur le plan touristique (hôtels), socio-économique (Salon international d'exposition) et l'implantation, prochainement, d'une ville verte. Toutes ces constructions sont faites aux dépens de la forêt et de la plage de Haouzia. En outre, cette plage attire plus d'estivants malgré l'absence d'une infrastructure adéquate, ce qui favorise la prolifération des accès hasardeux à la plage et des parkings clandestins qui causent le déboisement de la forêt et à la fragilisation de la dune. Ces constatations ne sont pas isolées étant donné qu'au SW d'El Jadida d'autres traces de l'action anthropique ont été enregistrées (Ouadia M. et al., 2003 et 2005).

\section{Conclusion}

De l'étude de la plage de Haouzia ressort qu'il s'agit d'une plage ample, plus ou moins homogène et rectiligne avec un peu plus de déferlement des vagues au SW et que l'influence de l'embouchure de l'Oued Oum Rbia au NE est significative.

L’hétérogénéité des profils et leur variation spatiotemporelle sont dues, certainement, à l'interaction dynamiques marine/dynamique éolienne et la morphologie de la plage.

Les taux, généralement, moyens en carbonates, prouvent la participation des calcaires crétacés (en amont d’Azemmour) et des calcarénites quaternaires (substratum) à l'alimentation des sédiments de la plage de Haouzia. L'apport des sédiments terrigènes par l'Oued Oum Rbia participe à la dilution de ces sédiments et par conséquent à la diminution du taux des carbonates. L'hétérogénéité des sédiments, en particulier, au SW de la plage mais aussi dans l'estran témoigne du déferlement des vagues et de l’irrégularité de la dynamique marine en hiver grâce à des tempêtes. 
La texture fine au SW et en été s'explique par la faible dynamique marine et dans les dunes par la nature de l'agent du transport sélectif (vent). En général, les sédiments sont bien classés (valeurs faibles de So) que ce soit les sédiments grossiers ou fins $(1<\mathrm{Sk}<1)$. Le maximum du classement est celui des sédiments de la dune à cause du tri sélectif.

Les minéraux lourds des sédiments de la plage de Haouzia montrent que les sources émettrices sont, en particulier, les terrains métamorphiques de Boulaouane et ceux magmatiques à Sidi Said Maachou. Leurs principaux minéraux (pyroxène, micas et disthène) sont acheminés par l'Oued Oum Rbia jusqu'à son embouchure à Azemmour puis repris par la dérive littorale vers la plage de Haouzia.

La teneur pondérale des minéraux lourds, élevée au NE, témoigne de l'apport de ces derniers par l'Oued Oum Rbia.

La plage de Haouzia subit une pression anthropique non négligeable. Celle-ci réside dans la prolifération des unités touristiques mais aussi dans le déboisement de la forêt. Si aucune intervention n'est faite pour limiter ces activités, la plage de Haouzia connaîtrait des conséquences irréversibles d'où une sensibilisation et une protection de cette plage s'impose urgemment.

\section{References:}

ABERKAN M., ABOUMARIA Kh. et OUADIA M., (2014) - Apport de l'observation du paysage côtier actuel et de l'organisation des séquences littorales sur la paléogéographie du Pléistocène récent au Nord-Ouest du Maroc. Actes RQM6, Tanger, pp. 79-85

ABERKAN M., ABOUMARIA Kh., ZAGHLOUL M.N. et OUADIA M., (2015) - Dynamique littorale et instabilité des formations quaternaires de la façade atlantique marocaine : Impact sur l'aménagement de la zone costière entre Rabat et Larache (Maroc). RQM8, Nador, pp. 39-40.

ABOUMARIA K., 1993. Les formations quaternaires du Sahel des Doukkala (Meseta occidentale marocaine) : mise en place et évolution post-sédimentaire. Thèse Doctorat 3ème cycle, Univ. Mohammed V, Fac. Sci., Rabat, 186 p.

AKIL M. (1990) - Les dépôts quaternaires littoraux entre Casablanca et Cap Beddouza (Méséta côtière marocaine) - Etudes géomorphologiques et sédimentologiques. Thèse Doc. Es., Fac. Sci, Rabat, 417p.

CHAIBI M., 2003. Dynamique sédimentaire et morphogenèse actuelle du littoral d'El Jadida (Maroc). Thèse Doctorat Es-sciences, Univ. AixMarseille, $235 \mathrm{p}$.

CHAIBI M. et Sabatier F., 2010-Budget sédimentaire du littoral de la baie d’El Jadida (Maroc). Méditerranée, 115|2010: Rivages p. 116124méditerranéens. 
KHOUAJA S., OUADIA M., ECH-CHOURFY A. et IRZAN El. (2016) a - L'érosion de la falaise côtière de Haouzia : évaluation et évolution spatiotemporelle. Afrique SCIENCE, Février 2016, p. 222- 234.

KHOUAJA S., OUADIA M. et IRZAN El. (2016) b- Fonctionnement de la plage d'El Jadida (Côte atlantique marocaine) : apport de l'étude morphodynamique, sédimentologique, minéralogique et de l'action anthropique ». ESJ, Juin 2016, Vol.12, No.18, p. 93- 106.

MHAMMDI N., ACHAB M, HAMOUMI N. et AZZA A. (2005) - Les sables titanifères du littoral d'Azemmour et de l'estuaire de l'Oum Er-Rbia (côte atlantique marocaine) : sédimentologie et potentiel d'exploitation. Bulletin de l’Institut Scientifique, section Sciences de la Terre, 2005, n²7, 83-91.

OUADIA M. (1998)- Les formations plioquaternaires dans le domaine mésétien occidental ente Casablanca et Safi : Géomorphologie, Sédimentologie, Paléoenvironnements quaternaires et Evolution actuelle, Thèse Doct. d'Etat, Univ. Mohammed V, Rabat, Maroc 319 p.

OUADIA M. et ABERKAN M. (2003) - Influence des actions naturelle et anthropique sur la Méséta occidentale marocaine (entre El Jadida et Safi). Actes du colloque : Les $2^{\text {ème }}$ journées des Géosciences de l'Environnement, Kénitra, pp. 1-7.

OUADIA M., ABERKAN M., ABOUMARIA Kh. et NISSOUL A. (2005) Action anthropique dans la Méséta côtière marocaine (Entre Casablanca et Safi) : depuis le Pléistocène supérieur jusqu'à l'Actuel., 3ème Rencontre des quaternaristes marocains, Marrakech, p. 47.

OUADIA M., ABERKAN M. et ABOUMARIA K. (2015) - Le complexe dunaire quaternaire du Jorf Lasfar (Meseta côtière occidentale marocaine) : lithostratigraphie, sédimentologie et néotectonique. Paralia, Vol. 8, $\mathrm{N}^{\circ}$. 1, pp n02.1-n02.14, DOI: http://dx.doi.org/10.5150/revue-paralia.2015.n02

PARFENOFF A., POMEROL C. et TOURENG J. (1970) - Les minéraux en grains : méthodes d'étude et détermination. Masson et Cie Ed., Paris, 499 p. VATAN A. (1967)- Manuel de sédimontologie.Technip Ed. Paris 397 p.

ZOURARAH B. (2002) Les processus côtiers actuels et leur impact sur l'environnement littoral des Doukkala (côte atlantique marocaine): Approche hydrodynamique, morphologique. sédimentologique et géochimique. Thèse Doctorat, d'Etat. 